\title{
Peptidic Alginate-Based Hydrogels Demonstrate Chemotaxis and Expansion to Adipose Tissue Derived and Blood Derived Stem Cells
}

\author{
E. Russell Vickers \\ Clinical Stem Cells PL, Sydney, Australia \\ Email: manager@clinicalstemcells.com
}

How to cite this paper: Vickers, E.R. (2020) Peptidic Alginate-Based Hydrogels Demonstrate Chemotaxis and Expansion to Adipose Tissue Derived and Blood Derived Stem Cells. Journal of Biomaterials and Nanobiotechnology, 11, 245-259. https://doi.org/10.4236/bnb.2020.114016

Received: August 12, 2020

Accepted: September 15, 2020

Published: September 18, 2020

Copyright $\odot 2020$ by author(s) and Scientific Research Publishing Inc. This work is licensed under the Creative Commons Attribution International License (CC BY 4.0).

http://creativecommons.org/licenses/by/4.0/

\section{(c) (i) Open Access}

\begin{abstract}
Biodegradable scaffolds have a major therapeutic advantage in regenerative medicine with their ability to include multiple compounds of drugs, growth factors and more recently, stem cells within the matrix. The scaffold can be programmed with mechanoresistive parameters targeted to the tissue to be replaced. Direct chemoattraction of in vivo stem cells to the implanted scaffold would be advantageous in the clinical setting. Large peptides such as vasculo-endothelial growth factor have demonstrated chemotaxis for angiogenesis from endothelial cells. This suggests other endogenous peptides may be present to directly attract stem cells to a scaffold. This exploratory study assessed if peptides from the blood peptidome would display chemotaxis to stem cells. Results showed that several short N-mer peptides demonstrated remarkable chemotaxis to blood and adipose tissue derived stem cells. Sodium alginate hydrogel was placed into 6-well, 24-well plate, and partitioned plates with channels between the wells. Connected wells were in series and spiked with peptides, biofluids containing stem cells and control wells. Images were recorded between three and nine days after incubation at $37^{\circ} \mathrm{C}$. There were rapid migration and expansion of stem cells into the peptide wells. Cell analysis revealed activated stem cells on a number of parameters including autophagy, Ki67 and nitric oxide. Potentially, this enhanced method to bioscaffold design utilizing peptide chemoattraction could result in an improved approach for stem cell therapy and regenerative medicine applications. Specific patient groups (e.g. blood coagulation disorders) where surgery to acquire adipose tissue or bone marrow is contraindicated may benefit. In addition, the technology is portable and safe by using "on demand" peripheral blood derived stem cells and would be particularly suitable for specialized environments such as space medicine.
\end{abstract}




\section{Keywords}

Chemoattraction, Chemotaxis, Peptides, Stem Cells, Hydrogel, Alginate, Space Medicine

\section{Introduction}

Comprehensive tissue engineering encompasses diverse and developing fields that include biological scaffolds, drug delivery systems, nanoengineering and optimizing the extracellular matrix for cell signaling. Detailed reviews of the scope of this area have been recently reported [1] [2]. An area of great interest has been seeding of the scaffold to enhance various mechanistic aspects of the framework for stem cell differentiation. Direct seeding of stem cells into the scaffold however has been problematic due to issues of maintaining cell viability during the laboratory manufacturing process and maintaining cell survival when transplanted into the animal or human. In addition, the construct must retain sufficient mechanoresistive properties to maintain the 3D structure while allowing permeation of critical molecules for cell survival and function. Biodegradable microneedles have been explored for transdermal drug delivery systems with the outer coating and needle point comprised of natural compound materials such as crystalline hyaluronic acid, with the inner chamber housing the therapeutic drug [3]. One further novel concept has been impregnating a biodegradable 3D printed outer polylactic-glycolic acid shell enveloping a gel housing mesenchymal stem cells, specifically for skin regeneration [4]. However, the researchers commented that the approach can only contain a minimal number of stem cells.

One of the original scaffolds to be tested for regenerative medicine is sodium alginate derived from seaweed. This has undergone significant research by cardiac regenerative medicine specialists [5]. Interestingly, the material has had a very long history of use in dentistry with its principal application for recording the impression of teeth and oral mucosa for the construction of dentures. The two forms were the thermally reversible alginic acid hydrocolloid that could be reused and the irreversible sodium alginate crosslinked version. Seaweed forms part of the diet of several Asian countries and supports its use as a safe material for human in vivo applications.

The incorporation into the scaffolds of multiple constituents that provide nutritional support, and other compounds for cell signaling can promote stem cell expansion and differentiation. This is a developing field in order to optimize the scaffolds prior to surgical insertion. Cytokines, growth factors, proteins and their subunit peptide fractions are being investigated. Background research on these compounds has largely focused on angiogenesis to make a viable scaffold. Angiogenesis is the development of new blood vessels from pre-existing vessels by sprouting and splitting and the process is vital for successful tissue and organ viability. Endothelial cells, pericytes and fibroblasts can be directed by molecular 
chemoattractant cues from the protein vasculo-endothelial growth factor (VEGF) [6] [7] [8]. VEGF functions are multiple and include chemotactic signaling, endothelial polarization, VEGF-induced cell migration, and endothelial tube formation. However, the protein is also a key regulator for cancer cell invasion into tissues [9]. It has been recently shown that fibroblast growth factor 2 (FGF-2) derived conditioned medium from the stem cell expression proteome also displays chemoattractant ability to induce angiogenesis [10]. Other peptidic compounds from blood have being investigated for their respective potential in immune cell chemoattraction, for example, chemoattraction of macrophages, $\mathrm{T}$ lymphocytes, mast cells and dendrites to $\alpha$-defensin peptide [11].

Recently, peptide inclusion with embryonic stem cells for specific cell differentiation within the scaffold has been investigated for cardiac regeneration. Cardiac stem cells are highly specialised and circulating stem cells do not have the ability to form myocardium as a result of hypoxic tissue damage [12]. However, hematopoietic or adipose stem cells are very useful for differentiation where there is injured nerve tissue or bone trauma. For this application other specific neuropeptides such as nerve growth factor, or bone morphogenetic protein for osteoblasts could be included in the bioframework. Stem cell viability within the scaffold is still in preliminary stages of investigation and is a rate limiting step at present. A logical development for bioscaffold architecture would be identification of hematopoietic based peptides that attract local niche stem cells and possibly circulating or adipose stem cells to the scaffold via a chemical gradient (chemotaxis). This would enhance the laboratory placement of stem cell depots within an implanted scaffold.

Blood has an extensive cellular and biochemical makeup. In particular, its biochemistry regarding peptides has phenomenal complexity. Recent triple quadrupole mass spectrometric analysis (LC/MS/MS) of blood revealed an astounding 1,916,672 distinct best-fit peptide correlations from 15,968,550 MS/MS spectra [13]. To identify candidates of stem cell chemotactic peptides from the milieu of biofuids would present extraordinary difficulty regarding time and cost associated with the likelihood of successful translational clinical outcomes. Two well studied blood proteins for hematopoietic stem cell migration are macrophage colony stimulating factor 1 (CSF-1, 522 amino acids) and stromal derived factor 1 (SDF-1, 89 amino acids) [14] [15]. However, CSF-1 and SDF-1 have long sequences and are costly to synthesise for clinical research.

The purpose of this study was to identify short sequence candidate peptides that could potentially demonstrate chemotaxis to circulating hematopoietic and adipose derived stem cells. Selected peptide sequences were based on unpublished data by the author from previous MS analyses of biofluids. These sequences were compared to the MS/MS results as previously mentioned [13]. Peptides were synthesised and then added to an alginate hydrogel to observe chemotaxis and expansion of stem cells for confirmation. Preliminary flow cytometry and cell analysis was performed comparing fresh adipose derived stem cells (ADSCs) from the stromal vascular fraction (SVF), migrated and expanded 
ADSCs from the peptide hydrogel, and migrated and expanded hematopoietic stem cells (HSCs) from peripheral blood placed in the peptide hydrogel.

\section{Methods}

\subsection{Stem Cells}

ADSCs were acquired from residual tumescent lipoaspirate following medical procedures for fat removal on consenting adults (three males and five females, age range $30-78$ years, mean 59.1 years \pm SD 15.7). The tissue was mechanically emulsified, vortexed and centrifuged to yield the cell pellet. Cells were plated between 12 and 48 hours after collection and stored in Eppendorf tubes at $37^{\circ} \mathrm{C}$. Two alginate concentrations were tested of $3 \%$ and $5 \%$ with $2.9 \%$ glutamine. Incubation was performed at $37^{\circ} \mathrm{C}$. Circulating HSCs were obtained from a 50ul sample of whole blood drawn by a diabetic needle applied the fingertip from a healthy 65 year old male with no significant medical conditions and not taking medication.

The method to assess stem cell migration was adapted from the EMD-Millipore application notes using tissue culture wells. Well plates (BD Biosciences) were 6-well, 12-well and 24-well plates with wells connected by a $2-3 \mathrm{~mm}$ borehole. $8 \mathrm{ml}$ of gel was deposited to each well in the 6-well plate, and $5 \mathrm{ml}$ of gel to the 12- and 24-well plate. Additional two and four compartment partitioned $80 \mathrm{~cm}$ plastic Petri plates assessed relative chemotactic potency of the peptides in some of the assays.

\subsection{Peptides}

Peptides were synthesised by standard F-moc solid state synthesis protocols. Confirmation and purity (>99\%) of peptides were performed by liquid chromatography coupled to a single quadrupole mass spectrometer (Shimadzu LCMS). Candidate peptides were of short sequence $(<20$ mer). A total of 15 analytes (numbered P1 to P15) were synthesised from the theoretical list of $>500$ peptides considered by the author as having potential chemoattraction. P8 had a single disulphide bond and all remaining peptides were of linear sequence.

Preliminary testing of the dispersion of peptides was assessed by methylene blue spiked into a control gel (Figure 1). Peptides were added to the target well at

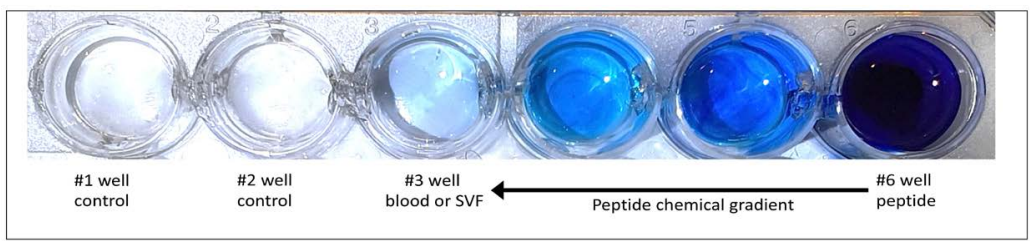

Figure 1. Image of well design to indicate peptide dispersion to form the chemical gradient at 24 hours, row 1 of a 24 well plate. All wells connected by perforations in the side walls. Methylene blue dye $1 \mathrm{mg}$ used as the test agent and placed in well $\# 6.5 \mathrm{ml}$ of the $3 \%$ alginate hydrogel deposited in each well. For peptide chemotaxis experiments wells \#1 \& \#2 were control gels, \#3 used as the well for placing blood or stromal vascular fraction, well \#6 for peptide placement. 
a concentration of 1 - $5 \mathrm{mg}$ for each analyte. The well configuration had the biofluid located in the central alginate well, with adjacent wells containing peptide in gel, and control gel. All peptides were tested twice with further repeat experiments conducted on peptide gels demonstrating chemoattraction to stem cells by the development of colony forming units (CFUs) at the macroscopic level.

\subsection{Imaging}

Macroscopic images were recorded by the magnifier app on a Samsung Note 10 cell phone. Low resolution images $(<50 \times)$ were made by a USB camera with software (Dinolite, Taiwan). Higher resolution images $(>100 \times)$ were made a Nikon inverted microscope. Images were recorded at baseline, and between 3 days to 9 days after plating. Images were cropped, and then adjusted with brightness and contrast to improve the stem cell colony forming units (CFUs) visibility.

\subsection{Flow Cytometry}

For cell analysis, CFUs in the gel were transferred to dual $20 \mathrm{ml}$ Luer lock syringes, phosphate buffered saline added for a working volume, then manually passaged through a $4 \mathrm{~mm}$ double ended female connector to reduce gel viscosity, then centrifuged at 1500 RPM for four minutes. The upper layer was decanted from the cell pellet and the procedure repeated three times to remove the gel. Trypsin $0.05 \%$ was added at the last cycle to dissociate the CFUs. Preliminary confirmation of stem cell identification used a Muse ${ }^{\oplus}$ Cell Analyzer (EMD Millipore Corporation, Hayward, CA, USA). The Muse has a forward scatter detector, a 532-nm green excitation laser with 576-nm yellow and 680-nm red emission detectors. The detection CD antibodies kit included CD 90, 45, 14, 34 (Muse Stem Cell Mesenchymal kit). The MUSE has additional screening parameters for cell chemistry and health parameters including autophagy induction (LC3), annexin V, EGFR expression, Ki67 proliferation, MAPK expression, H2A.X expression, nitric oxide (NO) and reactive oxygen species (ROS).

\section{Results}

Preliminary workup showed the chemical gradient approach was feasible using channels in multi-well plates (Figure 1). This permitted stem cells to migrate to the active test peptides and away from the control wells. The use of standard tissue culture apparatus of 6-well and 24-well plates with perforated walls allowed a chemical gradient to be established for preliminary validation of this method.

The 3\% and 5\% alginate hydrogels showed rapid expansion of cells to confirm that macroscopic imaging was possible (Figure 2, Figure 3). CFUs were clearly observed in the hydrogels. Both 2D gel surface expansion and deeper 3D colonies were seen.

In this screening study six analytes were identified with chemoattractant properties to HSCs and ADSCs. Peptides P1 and P2 with high sequence homology of $70 \%$ showed only minimal chemotaxis when individually tested, but chemoactivity was greatly enhanced when combined together (Figure 4). 


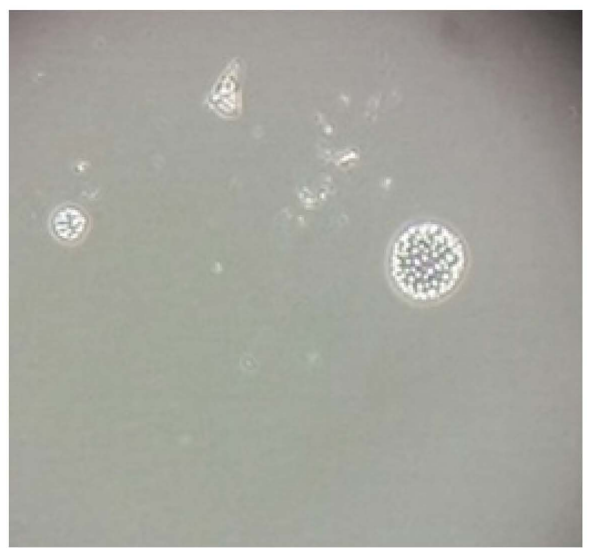

(a)

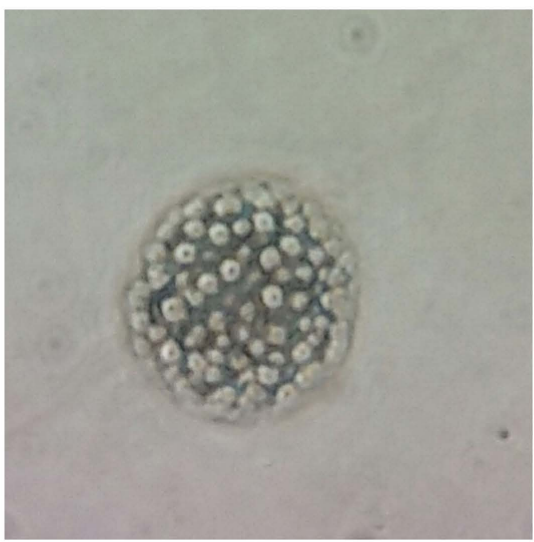

(b)

Figure 2. Adipose stem cell colony expansion in 5\% alginate hydrogel to evaluate stem cell expansion capability of the hydrogel, without peptides. (a) and (b) images under low and high magnification, respectively of developing colonies in gel at 7 days.

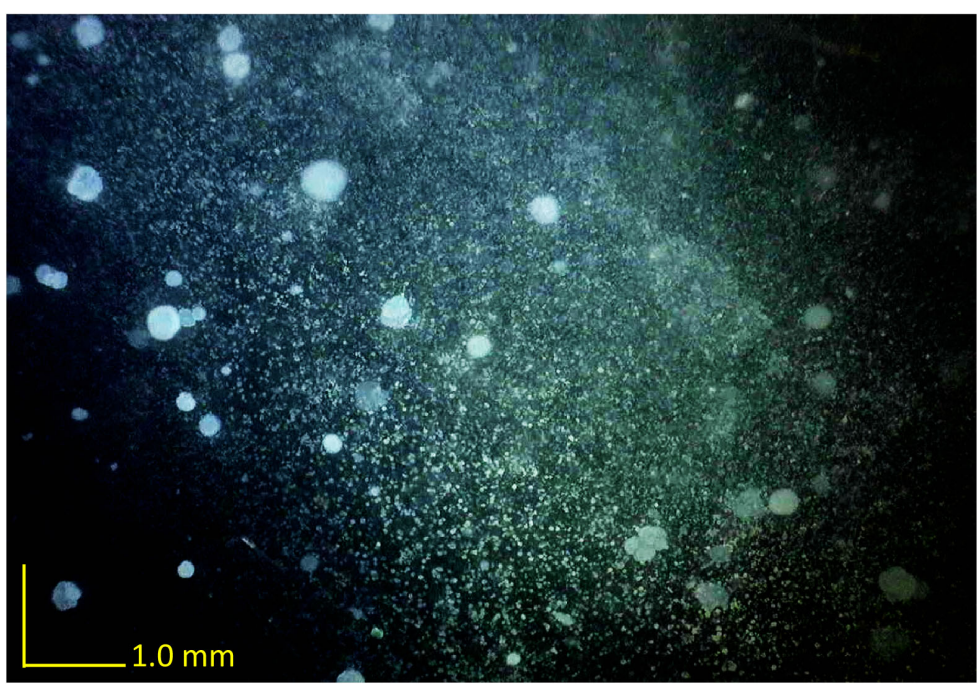

Figure 3. Variable sizes of the stem cell colony forming units (CFUs) at 3 days in $5 \%$ alginate hydrogel without peptides. Image capturing at $10 \times-50 \times$ with the USB microscope was considered satisfactory for detecting separate colonies in peptide hydrogels.

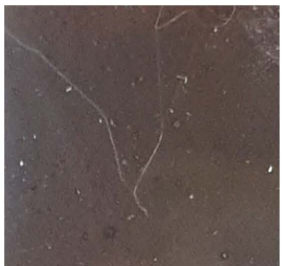

(a)

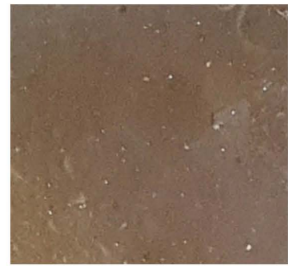

(b)

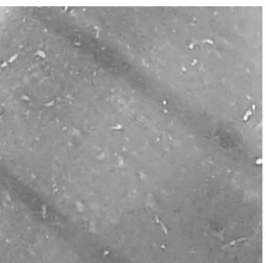

(c)

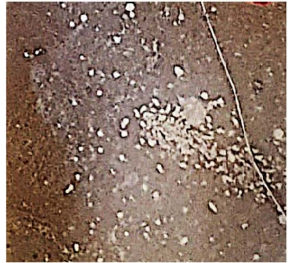

(d)

Figure 4. Chemotaxis comparison of adipose stem cells (ADSCs) in the stromal vascular fraction (SVF) to P1 and P2 peptides in a four partitioned Petri dish containing $3 \%$ alginate hydrogel at low magnification. The SVF was placed in the middle of the dish. Images captured at 4 days. (a) Control segment; (b) P1 peptide segment; (c) P2 peptide segment; (d) P1 and P2 peptide combined segment. (d) shows preferential migration and expansion of stem cell colonies with the combination of P1 and P2. 
Peptides P3, P4 and P5 also demonstrated chemoattraction to HSCs and ADSCs compared with controls (Figures 5-7). Peptide P3 demonstrated superior chemoactivity compared with several the other peptides (Figure 8). Analytes P8, P9 and P10 also demonstrated strong chemoattractant properties and significant development of CFUs from ADSCs and HSCs (Figure 9).

\section{Analysis of migrated expanded cells}

The stem cell colonies were positive for CD90/45. A comparison of the freshly acquired ADSCs, migrated/expanded ADSCs, and migrated/expanded HSCs showed activation on these cells for autophagy induction (LC3), EGFR expression, Ki67 proliferation, P13, NO, ROS and RNA content (Figure 10, Table 1).
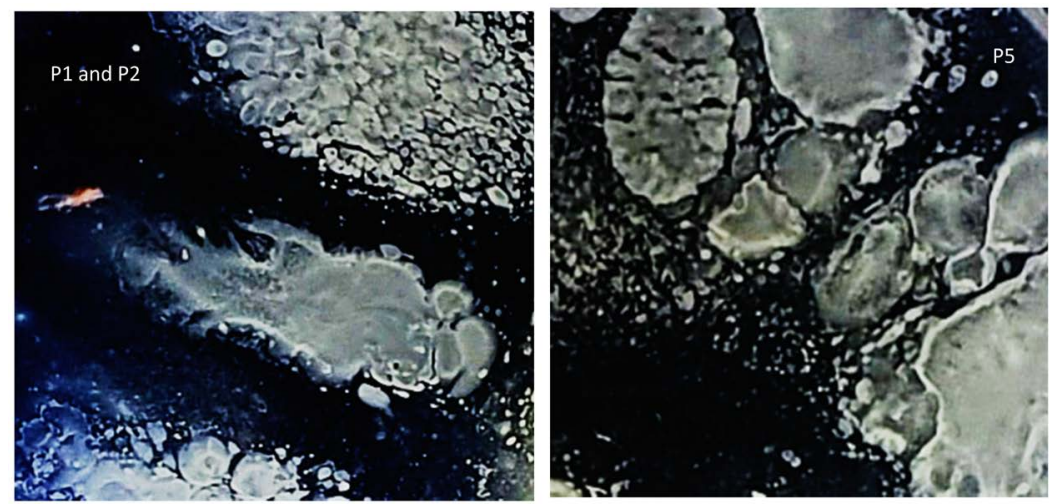

Figure 5. Comparative potency of peptide chemotaxis to hematopoietic stem cells. $50 \mathrm{uL}$ blood placed in the middle of a two partitioned $80 \mathrm{~cm}$ Petri dish containing $5 \%$ alginate hydrogel. The halves comprised of combined P1 \& P2 (left) versus P5 (right). Macroscopic images taken at 5 days after plating. Density of the stem cell colonies showed equal chemotaxis.
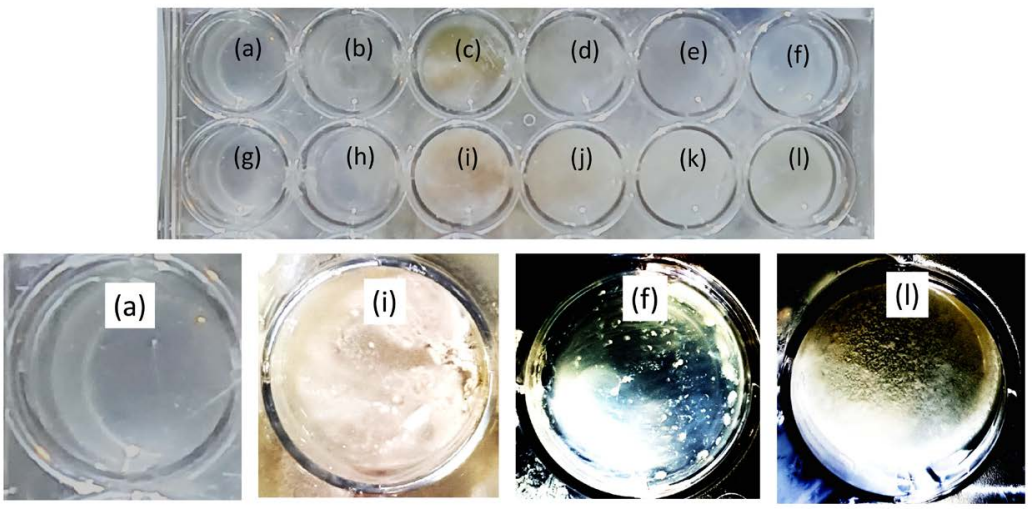

Figure 6. Chemotaxis assay for P3 and P4 peptides in 3\% alginate hydrogel for hematopoietic stem cells. $5 \mathrm{ml}$ of gel in each well. Middle two rows of 24-well plate used and channels between each well for each row. Images taken at 6 days after plating with low magnification. Row (a)-(f) assessed P4 peptide: (a), (b) control wells; (c) 50 uL blood; (d)-(f) wells containing increasing gradient of P4 with F being the spiked well of P4. Images show no colonies in the (a) or (b) control wells, significant stem cell colonies seen in (d)-(f). Row (g)-(l) assessed P3 peptide: (g), (h) control wells; (i) 50 uL blood; (j)-(l) wells containing increasing gradient of P3 with $\mathrm{L}$ being the spiked well of P3. Images show no colonies in the (g) or (h) control wells, significant stem cell colonies seen in (j)-(l). 


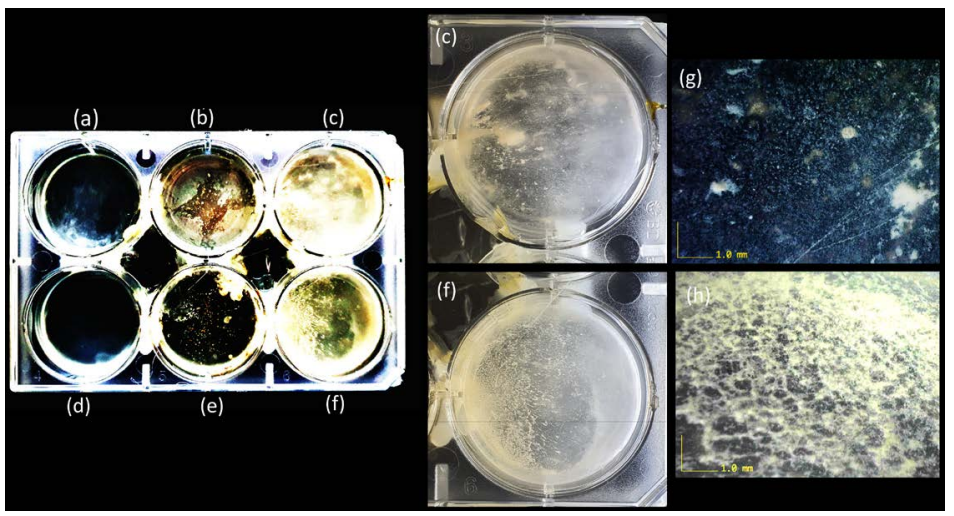

Figure 7. Chemotaxis assay for P1 and P2, and P3 peptides in 3\% alginate hydrogel for adipose stem cells within the stromal vascular fraction (SVF). $8 \mathrm{ml}$ of gel in each well of a 6-well plate. Channels between each well for each row. Images taken at 9 days after plating with low and medium magnification. Row (a)-(c) assessed P3 peptide: (a) control well; (b) 50 ul SVF; (d) well containing spiked sample of P3 peptide. Images show no colonies in the control well (a), and significant stem cell colonies (c) the P3 spiked well with higher magnification image captured with USB microcsope and $1 \mathrm{~mm}$ measurement bar in image (g). Row (d)-(f) assessed combined peptides P1 and P2: (d) control well; (e) $50 \mathrm{uL}$ stromal vascular fraction; (f) well containing spiked sample of P1 and P2 peptides. Images show no colonies in (d) the control well, and significant stem cell colonies in (f) the P1 and P2 spiked well with higher magnification image captured with USB microscope and 1 mm measurement bar in image (h). (a) control; (b) SVF; (C) P3; (d) control; (e) SVF; (f) P1 \& P2; (c) P3; (f) P1 \& P3; (g) P3; (h) P1 \& P2.

Table 1. Cell analysis summary. Comparison of (a) baseline ADSCs from SVF in a 42 year old female analysed at 30 minutes after liposuction; (b) ex vivo migrated and expanded ADSCs into P9 hydrogel at 5 days from a 46 year old female; (c) ex vivo migrated and expanded HSCs from peripheral blood into P10 hydrogel at 5 days from a 65 year old male.

\begin{tabular}{|c|c|c|c|}
\hline Cell Analysis Parameter & Baseline ADSCs & Peptide gel ADSCs & Peptide gel HSCs \\
\hline CD90/45 & Positive & Positive & Positive \\
\hline Annexin V apoptosis (live cells) & $98.99 \%$ & $92.4 \%$ & $98.75 \%$ \\
\hline Autophagy (LC3 intensity) & $\begin{array}{c}14.3 \\
\text { quiescence }\end{array}$ & $\begin{array}{c}28.9 \\
\text { activated }\end{array}$ & $\begin{array}{c}39.5 \\
\text { activated }\end{array}$ \\
\hline EGFR expression (activated) & $0.1 \%$ & $6.7 \%$ & $8.11 \%$ \\
\hline H2A.X expression (activated) & $0.8 \%$ & $18.82 \%$ & $18.82 \%$ \\
\hline Ki67 expression (positive) & $2.08 \%$ & $4.95 \%$ & $7.8 \%$ \\
\hline P38 MAPK expression (activated) & $1.4 \%$ & $15.94 \%$ & $14.97 \%$ \\
\hline Mitopotential (depolarized live) & $92.6 \%$ & $96.4 \%$ & $80.73 \%$ \\
\hline Nitric oxide profile (live positive) & $0.03 \%$ & $0.01 \%$ & $0.1 \%$ \\
\hline Reactive Oxygen Species (positive) & $0.07 \%$ & $0 \%$ & $0.94 \%$ \\
\hline P13 activation & $0.3 \%$ & $16.91 \%$ & $48.1 \%$ \\
\hline Smartflare RNA content profile (mean fluorescence intensity) & 8.3 & 68.8 & 69.9 \\
\hline
\end{tabular}

Description of Parameter Functions: 1) CD90/45 are positive markers for stem cells. 2) Autophagy plays critical roles in stem cell quiescence, activation, differentiation, and self-renewal. 3) EGFR expression shows increased phosphorylation during mitosis. 4) H2A.X is increased during promotion of self-renewal for stem cells. 5) Ki67 is a cell proliferation associated protein and is a marker distinguishing cycling from noncycling cells for growth potential. 6) P38 MAPK is the regenerative capacity of the stem cell and is upregulated in activated stem cells. 7) Mitopotential measures the depolarized membrane potential that facilitates reduced mRNA expression of senescence-associated markers, and increased mRNA expression of autophagy and immunomodulatory markers. 8) Nitric oxide is a critical signaling molecule that is required for stem cell mobilization and differentiation 9) Reactive Oxygen Species (ROS) has phases of low ROS level for quiescence and self-renewing stem cells; mild increase of ROS in stem cells causes lineage differentiation; high level ROS causes stem cell senescence or aging and cell death. 10) P13 (phosphoinositide 3-kinase) promotes hematopoietic stem cell activation and plays a crucial role for mitogenesis, proliferation, prevention of apoptosis, and maintenance of multipotency in mesenchymal stem cells. 11) Smartflare measures the release of small RNAs via exosomes for intercellular signalling and is related to cell origin and stemness. 

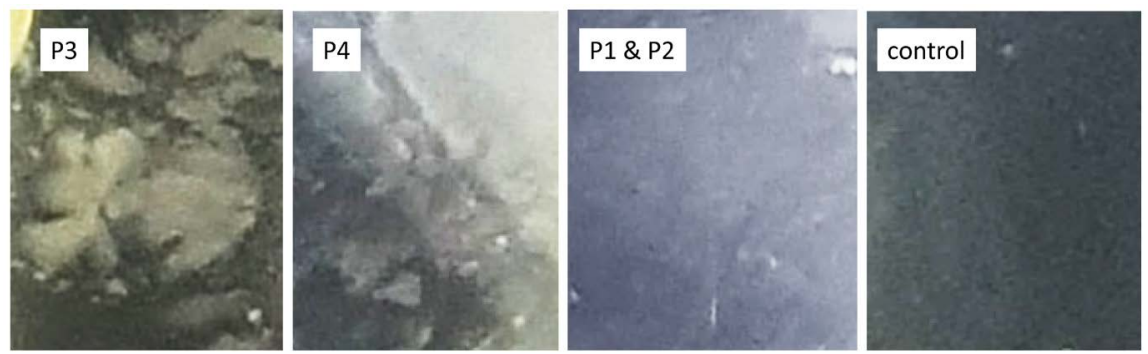

Figure 8. Comparative potency of peptide chemotaxis to the stromal vascular fraction (SVF) containing adipose stem cells. SVF placed in the middle of a four partitioned $80 \mathrm{~cm}$ Petri dish. The quarters comprised of P1 \& P2, P3, P4 and control. Density of the stem cell colonies showed chemotaxis of P3 > P4 > P1 \& P2 > control.

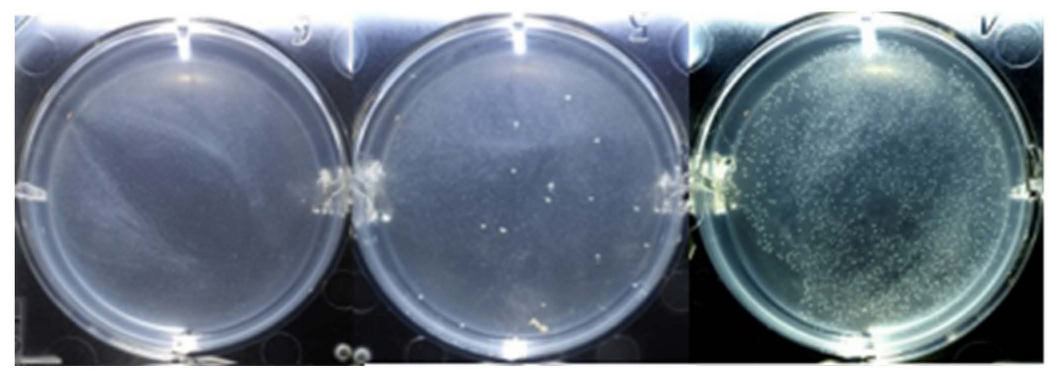

(a1)

(a2)

(a3)

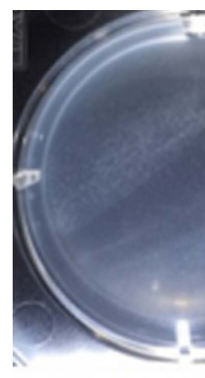

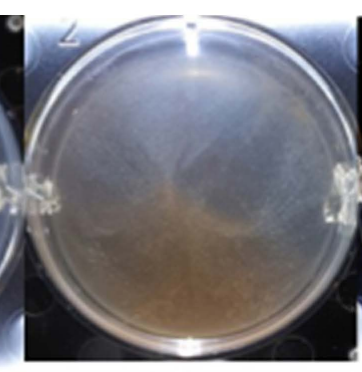

(b2)

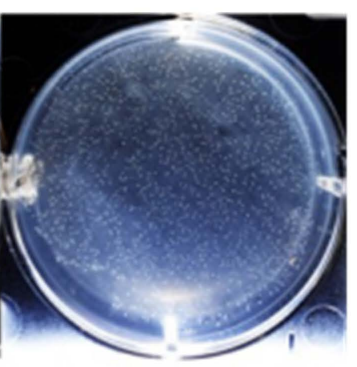

(b3)

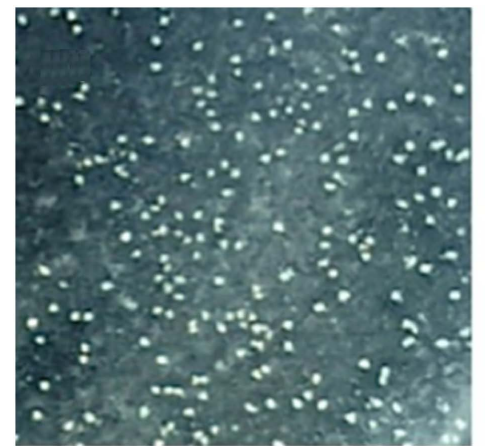

(a4)

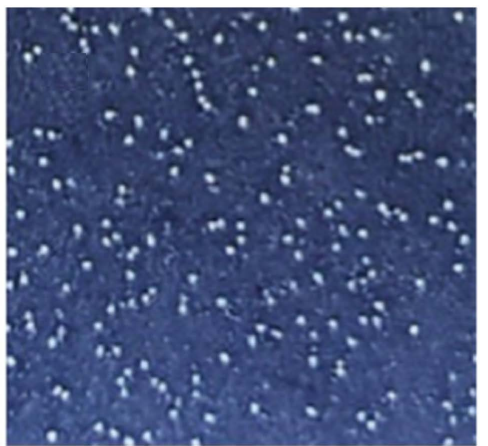

(b4)

Figure 9. Comparison of peptide gels spiked with ADSCs from the SVF and blood derived HSCs at 5 days incubation. (a) series is a 55 year old female; (b) series is a 65 year old male. (a1) and (b1) are control gels. (a2) is spiked with the SVF containing ADSCs and has 17 colony forming units (CFUs). (b2) is spiked with $50 \mathrm{ul}$ peripheral blood. (a3) and (b3) are P9 gels and have approximately 1500 CFUs and 1000 CFUs, respectively. (a4) and (b4) are magnifications of (a3) and (b3), respectively. (a1) control; (a2) SVF; (a3) CFUs peptide gel; (b1) control; (b2) blood; (b3) CFUs peptide gel. 
Column (a)

Baseline ADSCs

(a1)

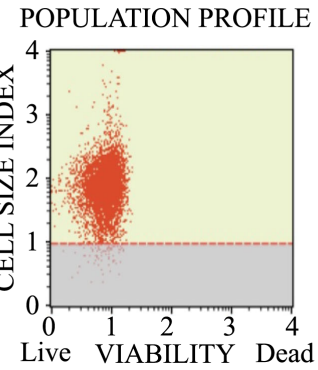

(a2)

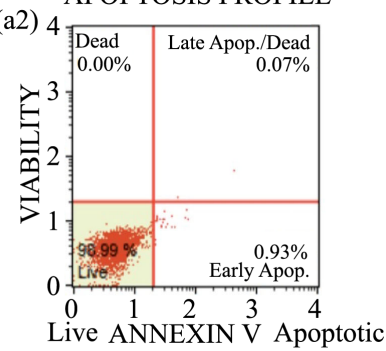

Column (b)

Peptide gel ADSCs

POPULATION PROFILE

(b1)

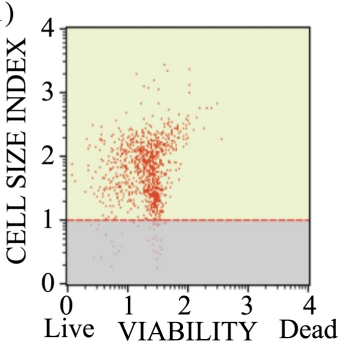

Live VIABILITY Dead

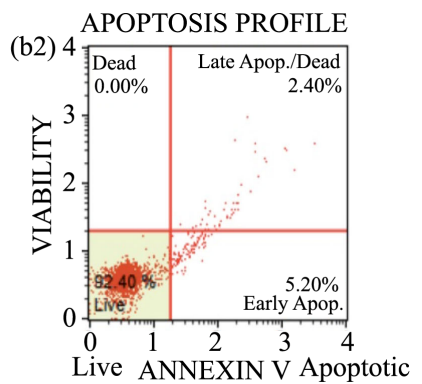

Column (c)

Peptide gel HSCs

POPULATION PROFILE

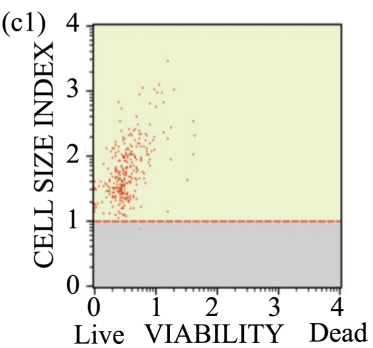

APOPTOSIS PROFILE

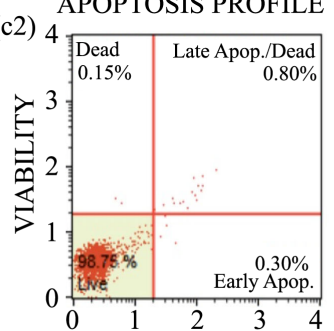

Live ANNEXIN V Apoptotic

(a3) AUTOPHAGY INDUCTION PROFILE (b3)AUTOPHAGY INDUCTION PROFILE (c3)AUTOPHAGY INDUCTION PROFILE

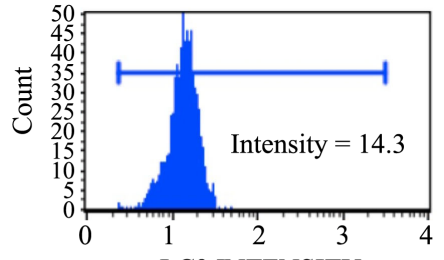

LC3 INTENSITY

(a4)

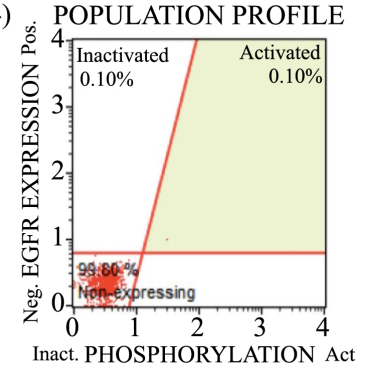

(a5)

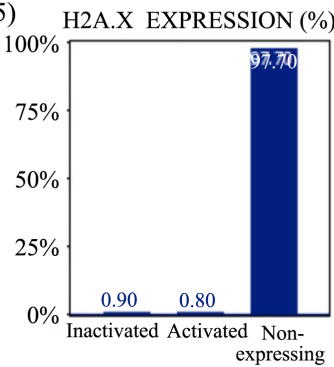

(a6) PROLIFERATION PROFILE

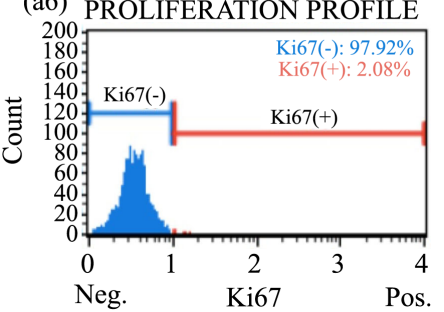

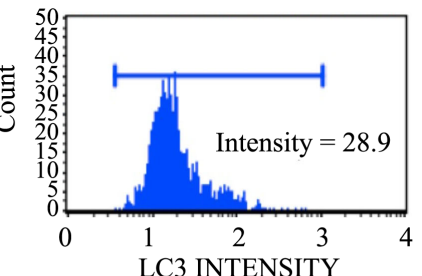

(b4)

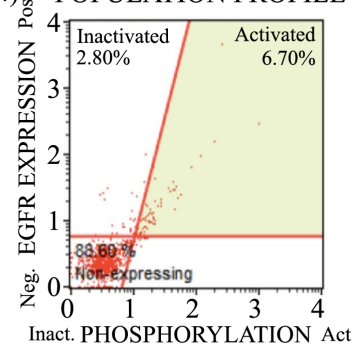

(b5) H2A.X EXPRESSION (\%)

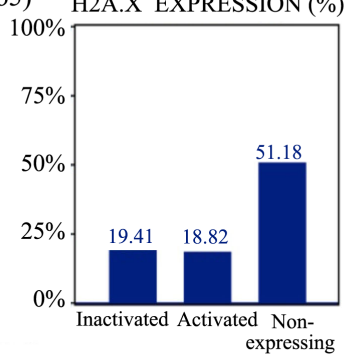

(b6)

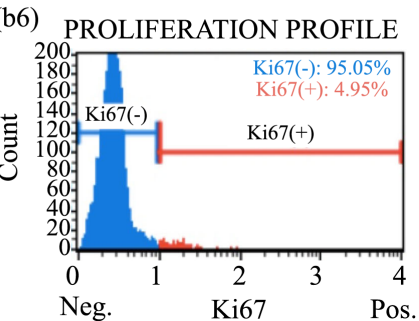

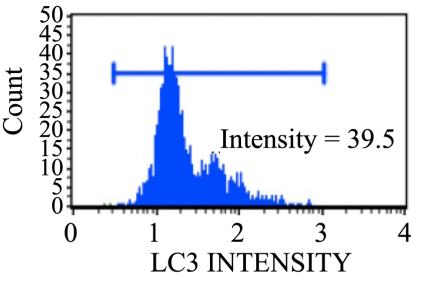

(c4)

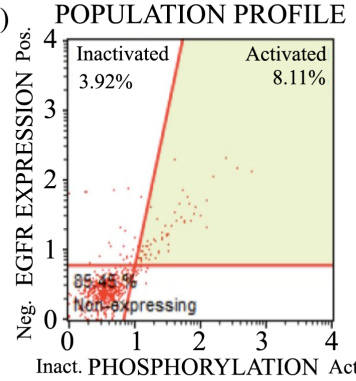

(c5) H2A.X EXPRESSION (\%)

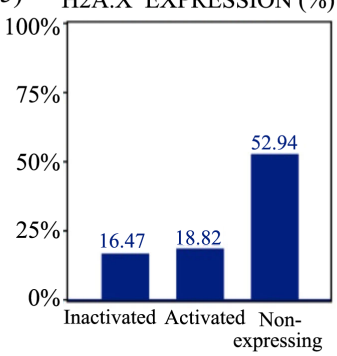

(c6) PROLIFERATION PROFILE

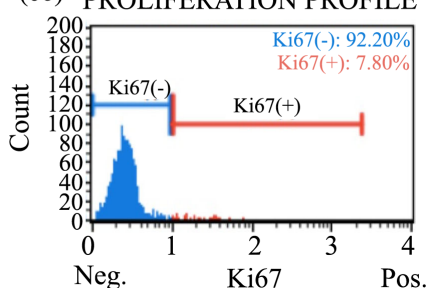




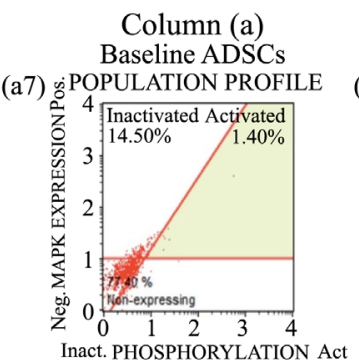

(a8) CELL HEALTH PROFILE

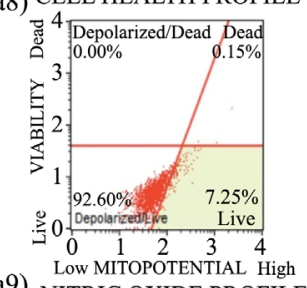

(a9) NITRIC OXIDE PR High

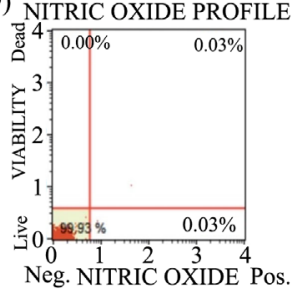

(a10)

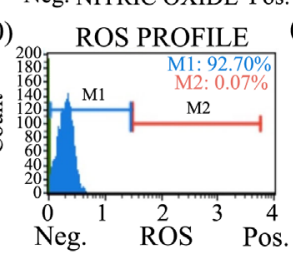

(a11) POPULATION PROFILE

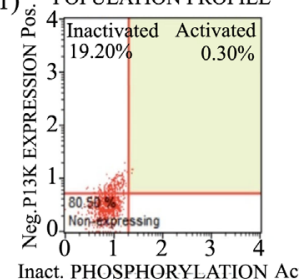

Inact. PHOSPHORYLATION Act

(a12)

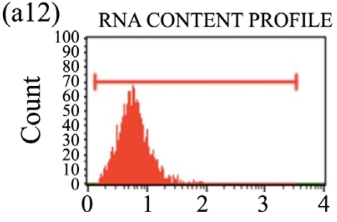

SMARTFLARE INTENSITY SMARTFLARE INTENSITY
Column (b) Peptide gel ADSCs

(b7) ¿्POPULATION PROFILE

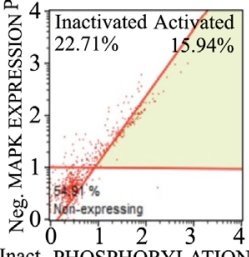

Inact. PHOSPHORYLATION Act

8) CELL HEALTH PROFILE

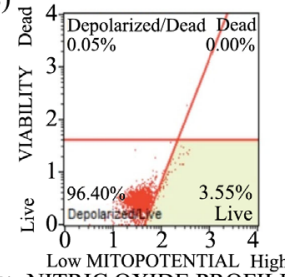

(b9)

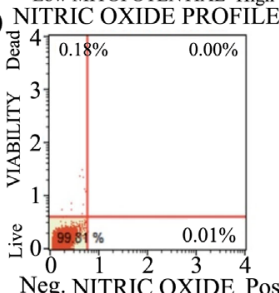

(b10)

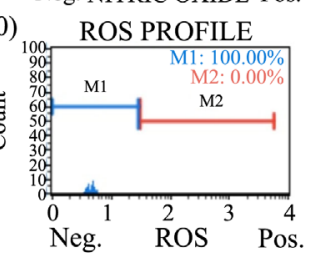

(b11)

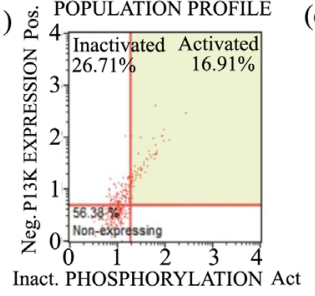

(b12) RNA CONTENT PROFILE

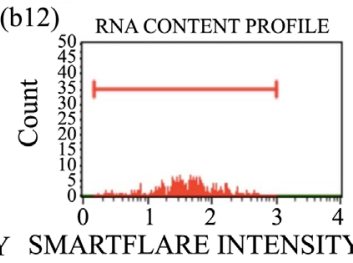

Column (c)

Peptide gel HSCs

(c7) $\stackrel{5}{\circ}$ POPULATION PROFILE

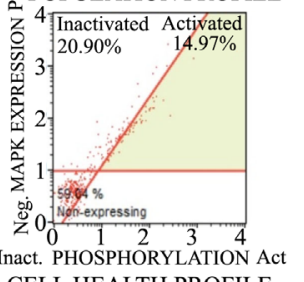

(c8) CELL HEALTH PROFILE

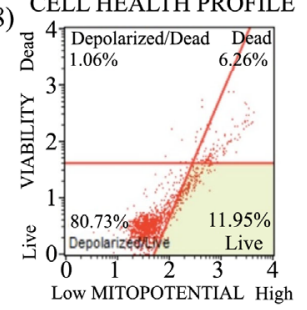

(c9) NITRIC OXIDE PROFILE

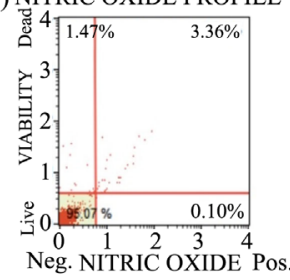

(c10)

ROS PROFILE

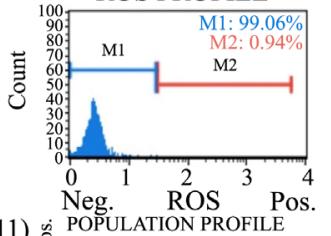

(c11)

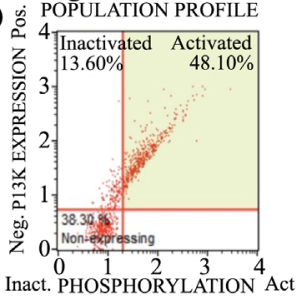

Figure 10. Cell analysis comparison from the Muse system. Comparison of (a) baseline adipose derived stem cells (ADSCs) from the stromal vascular fraction (SVF) from a 42 year old female; (b) ex vivo migrated and expanded ADSCs into P9 hydrogel at 5 days from a 46 year old female; (c) ex vivo migrated and expanded HSCs from peripheral blood into P10 hydrogel at 5 days from a 65 year old male. Column a represents baseline ADSCs from the SVF; Column b represents migrated and expanded ADSCs in peptide gel; Column c represents migrated and expanded HSCs in peptide gel. Row 1 CD90/45; Row 2 Annexin V; Row 3 Autophagy (LC3 intensity); Row 4 EGFR expression; Row 5 H2A.X; Row 6 Ki67 expression; Row 7 MAPK expression; Row 8 Mitopotential; Row 9 Nitric oxide profile; Row 10 Reactive Oxygen Species; Row 11 P13 activation; Row 12 Smartflare RNA content profile. 


\section{Discussion}

Macroscopic and low magnification images revealed differences in types of cell expansion. P1/P2 showed a preference for rapid 2D sheet formation on the surface of the gel, while P3 developed colony specific forms. Moreover, for P3 there was a variation in the rate of cell expansion in the colonies. This suggests that stem cells may have a hierarchical classification according to their role where some may have rapid migration and expansion for acute injury response, while others may have more dedicated tissue specific roles.

The use of the simplified well plate design was advantageous for this initial investigation and after reviewing methods for chemotaxis [16]. The most described method using Boyden cell filtration methodology culture separation was not considered due to assessing stem cell expansion for this study [17]. The use of the 24-well and 6-well plates in this current study was cost effective and permitted visual changes of chemotaxis and expansion to be readily assessed. The 24-hole plates can be designed in a number of configurations and depends on channel placement. Channels between wells were achieved by a fine pointed soldering iron pressed against the wall of the well. This perforated the wall and sealed the channel in a few seconds. The result was a simple, rapid and cost effective method.

This study identified several analytes for future consideration in chemoattractant bioscaffold design. One interesting finding was the dual use of P1 and P2 to initiate a strong chemotactic response, while individually the peptides exhibited minor chemotaxis. P1 and P2 are daughter peptides in the metabolic pathway of a single larger parent peptide. The blood peptidome is very extensive and it is likely there will be additional peptides with chemoactivity to stem cells. One advantage of identifying smaller $\mathrm{N}$-mer peptides is the rapid synthesis of mg quantities of the compounds by microwave peptide synthesisers. $\mathrm{N}$-acetylated peptides from synthesis offer a further advantage with a typically extended half-life for pharmacokinetic studies. The use of synthesised human endogenous peptides is another major advantage for clinical research and ultimately, for routine clinical use. Sequence homology between peptides showed $90 \%$ homology between P4 and P7, 70\% P1 and P2, and 33\% P9 and P10 but no sequence homology between other peptides. Single low mg dosing was used in the study and this was an effective dose for the in vitro experiments. Optimal dosing for each peptide is still required for in vivo use. The study primarily focused on ADSCs from the SVF. However, the inclusion of a sample of blood derived HSCs showed it was very effective in migrating and developing CFUs. Further research into this area is warranted, particularly with the ease of obtaining the blood and the prospect of having autologous stem cells "on demand" for specialized environments such as space medicine.

Sodium alginate hydrogels $3 \%$ and $5 \%$ were chosen for the gel matrix as cell viability and future safety issues have been published for its use in humans over the last 20 years. Alginate is widely available, used as a food additive and very 
economical to purchase for future laboratory and clinical research. Both $3 \%$ and $5 \%$ alginate concentrations were efficient for cell expansion. The $3 \%$ gel can be injected into tissue through an 18 gauge needle. This permits a straightforward pain free clinical use with prior placement of local anesthetic in a fine 27/30 gauge needle. Stronger mechanoresistive gels could be formed after injecting calcium or zinc crosslinking agents in a two phase delivery protocol. For example, a gel that is injected to suit the anatomical defect under CT image guidance such as bone cyst or endplate degeneration and then graded stiffness and compression can be measured by incremental dosing of the crosslinker in real time for the patient. Furthermore, there would be ample operative time available for surgeons using this approach. While there has been considerable development in sophisticated polycaprolactone scaffolds these materials can be difficult to adjust for viscosity by a surgeon where time is costly in the hospital operating theatre. One limitation of cell based crosslinked bioscaffolds constructed in the laboratory is difficulty in dissolving the scaffold to analyse cell morphology and health with CD markers and flow cytometry. Crosslinked alginate is a particularly difficult material to dissolve with non-toxic solvents after seeding of cells. Citrate buffer has been suggested but no validated method has been published. Cell analysis and cytometry on the non-crosslinked hydrogel in this study showed cell health was maintained in the migration and expansion of both ADSCs and HSCs. Applying a two stage approach of injecting the viscous stem cell non-crosslinked scaffold followed by a second injection of the cross linking agent would be feasible in clinical applications such as joint or nerve repairs.

Several clinical applications could be considered with a chemotactic peptide hydrogel without the inclusion of stem cells. Patients who may not be suitable for surgery to harvest adipose tissue stem cells are those with cancers, blood coagulation disorders, infectious patients and those taking immune suppressant drugs. In these situations a peptidic gel would be an advantage. In addition, developing stem cell organoids in a hydrogel that would actively attract the local resident stem cell niche may have improved outcomes for tissue differentiation and organ regeneration-for example, scars and burns, endocrine and exocrine organs, orthopaedic, ophthalmic, dental applications and nerve injuries. Neuropathic nerve pain is an example where ADSCs are safe and effective [18] but the described treatment employs harvested stem cells at a considerable operating cost. A simplified peptidic chemoattractant hydrogel as described in this study would be preferable for many patients with neuropathic medical conditions. The cost of this condition alone is immense with direct medical expenditure and lost productivity of neuropathic pain is estimated at USD $\$ 27,000$ per patient. With an estimated prevalence of $8 \%-10 \%$ of the US population the total figure gives a cost of over USD $\$ 700$ billion per year [19] [20]. Reducing the cost of the treatment with chemoattractant peptide hydrogels without the need for expensive stem cell tissue acquisition in an operating theater would be clearly beneficial for the health and biotechnology industry. 


\section{Conclusion}

Chemoattraction is frequently observed in nature at the macroscopic level such as butterfly pheromones and scavengers detecting cadaverine expression from rotting animal flesh. At the molecular and cellular level immune cells undergo rapid chemotaxis and migration to noxious by-products expressed by pathogens. This study shows that the blood proteome contains potent chemoactive peptides to elicit stem cell migration. When these peptides are placed into an alginate hydrogel there is recruitment and expansion of HSCs and ADSCs. This is a significant advancement for regenerative medicine therapy. It simplifies the clinical process and increases the safety margin for patients for numerous therapeutic interventions, particularly in patients where liposuction of adipose tissue is contraindicated. The endogenous peptides reported in this study are the first compounds identified to display intrinsic stem cell chemoattraction. It is likely there will be more active blood constituents with this property and opens a new area of research for regenerative medicine.

\section{Conflicts of Interest}

The author states that information from this study may be used for future commercial purposes.

\section{References}

[1] Nikolova, M.P. and Chavali, M.S. (2019) Recent Advances in Biomaterials for 3D Scaffolds: A Review. Bioactive Materials, 4, 271-292. https://doi.org/10.1016/j.bioactmat.2019.10.005

[2] Deb, P., Deoghare, A.B., Borah, A., Barua, E. and Lala, D.S. (2018) Scaffold Development Using Biomaterials: A Review. Materialstoday: Proceedings, 5, 12909-12919. https://doi.org/10.1016/j.matpr.2018.02.276

[3] Waghule, T., Singhvi, G., Dubey, S.K., Pandey, M.M., Gupta, G., Singh, M. and Duacde, K. (2019) Microneedles: A Smart Approach and Increasing Potential for Transdermal Drug Delivery System. Biomedicine and Pharmacotherapy, 109, 1249-1258. https://doi.org/10.1016/j.biopha.2018.10.078

[4] Lee, K., Xue, Y., Lee, J., Kim, H.-J., Liu, Y., Tebon, P., Sarikhani, E., et al. (2020) Biodegradable Polymers: A Patch of Detachable Hybrid Microneedle Depot for Localized Delivery of Mesenchymal Stem Cells in Regeneration Therapy. Advanced Functional Materials, 30, Article ID: 2070143. https://doi.org/10.1002/adfm.202070143

[5] Cattelan, G., Gerboles, A.G., Foresti, R., Pramstaller, P.P., Rossini, A., Miragoli, M. and Malvezzi, C.C. (2020) Alginate Formulations: Current Developments in the Race for Hydrogel-Based Cardiac Regeneration. Frontiers in Bioengineering and Biotechnology, 8, 414. https://doi.org/10.3389/fbioe.2020.00414

[6] Schor, A.M., Ellis, I. and Schor, S.L. (2001) Chemotaxis and Chemokinesis in 3D Macromolecular Matrices: Relevance to Angiogenesis. Methods in Molecular Medicine, 46, 163-183. https://doi.org/10.1385/1-59259-143-4:163

[7] Lamalice, L., Le Boeuf, F. and Huot J. (2007) Endothelial Cell Migration during Angiogenesis. Circulation, 100, 782-794. https://doi.org/10.1161/01.RES.0000259593.07661.1e 
[8] Evans, I.M, Kennedy, S.A., Paliashvili, K., Santra, T., Yamaji, M., Lovering, R.C., et al. (2016) Vascular Endothelial Growth Factor (VEGF) Promotes Assembly of the p130Cas Interactome to Drive Endothelial Chemotactic Signaling and Angiogenesis. Molecular and Cellular Proteomics, 16, 168-180.

https://doi.org/10.1074/mcp.M116.064428

[9] Schwab, M. (2011) Encyclopedia of Cancer. Springer, Berlin. https://doi.org/10.1007/978-3-642-16483-5

[10] Jin, S., Yang, C., Huang, J., Liu, L., Zhang, Y., Li, S., Zhang, L., et al. (2020) Conditioned Medium Derived from FGF-2-Modified GMSCs Enhances Migration and Angiogenesis of Human Umbilical Vein Endothelial Cells. Stem Cell Research and Therapy, 11, Article No. 68. https://doi.org/10.1186/s13287-020-1584-3

[11] Grigat, J., Soruri, A., Forssmann, U., Riggert, J. and Zwirner, J. (2007) Chemoattraction of Macrophages, T Lymphocytes, and Mast Cells Is Evolutionarily Conserved within the Human $\alpha$-Defensin Family. The Journal of Immunology, 179, 3958-3965. https://doi.org/10.4049/jimmunol.179.6.3958

[12] Hayoun-Neeman, D., Korover, N., Etzion, S., Ofir, R., Lichtenstein, R.G. and Cohen, S. (2019) Exploring Peptide-Functionalized Alginate Scaffolds for Engineering Cardiac Tissue from Human Embryonic Stem Cell-Derived Cardiomyocytes in Serum-Free Medium. Polymers Advanced Technologies, 30, 2493-2505. https://doi.org/10.1002/pat.4602

[13] Dufresne, J., Bowden, P., Thavarajah, T., Florentinus Mefailoski, A., Chen, Z.Z., Tucholska, M., Norzin, T., et al. (2018) The Plasma Peptidome. Clinical Proteomics, 15, Article No. 39. https://doi.org/10.1186/s12014-018-9211-3

[14] Markey, K.A. and Hill, G.R. (2017) Cytokines in Hematopoietic Stem Cell Transplantation, In: Foti, M. and Locati, M., Eds., Cytokine Effector Functions in Tissues, Academic Press, Cambridge, 219-236. https://doi.org/10.1016/B978-0-12-804214-4.00012-9

[15] Tang, T. and Hammond, H.K. (2015) Gene Transfer for Clinical Congestive Heart Failure. In: Laurence, J. and Franklin, M., Eds., Translating Gene Therapy for the Clinic. Techniques and Approaches, Academic Press, Cambridge, 215-226. https://doi.org/10.1016/B978-0-12-800563-7.00014-2

[16] Jin, T. and Hereld, D. (2009) Chemotaxis Methods and Protocols. In: Jin, T. and Hereld, D., Eds., Anticancer Research, Humana Press/Springer Science and Business Media, New York, 537. http://ar.iiarjournals.org/content/30/3/1035.2.full

[17] Chen, H.C. (2005) Boyden Chamber Assay. Methods in Molecular Biology, 294, $15-22$.

[18] Vickers, E.R., Karsten, E., Flood, J. and Lilischkis, R. (2014) A Preliminary Report on Stem Cell Therapy for Neuropathic Pain in Humans. Journal of Pain Research, 4, 255-263. https://doi.org/10.2147/JPR.S63361

[19] Schaefer, C., Sadosky, A., Mann, R., Daniel, S., Parsons, B., Tuchman, M., Anschel, A., Stacey, B.R., Nalamachu, S. and Nieshoff, E.C. (2014) Pain Severity and the Economic Burden of Neuropathic Pain in the United States: BEAT Neuropathic Pain Observational Study. ClinicoEconomics and Outcomes Research, 6, 483-496. https://doi.org/10.2147/CEOR.S63323

[20] Yawn, B.P., Wollan, P.C., Weingarten, T.N., Watson, J.C., Hooten, W.M. and Melton, L.J. (2009) The Prevalence of Neuropathic Pain: Clinical Evaluation Compared with Screening Tools in a Community Population. Pain Medicine, 10, 586-593. https://doi.org/10.1111/j.1526-4637.2009.00588.x 